\title{
Kerr-lens mode locked, synchronously pumped, ultra-broadband breathing pulse optical parametric oscillator Jintao Fan ${ }^{1,2}$, David Zuber ${ }^{1,2}$, Robin Mevert ${ }^{1,2}$, Tino Lang ${ }^{3}$, Thomas Binhammer ${ }^{4}$, Uwe Morgner ${ }^{1,2,5}$ \\ 1. Leibniz Universität Hannover, Welfengarten 1, 30167 Hannover, Germany \\ 2. Cluster of Excellence PhoenixD (Photonics, Optics, and Engineering-Innovation Across Disciplines), 30167, Hannover, Germany \\ 3. Deutsches Elektronen-Synchrotron DESY, Notkestraße 85, 22607 Hamburg, Germany \\ 4. neoLASE GmbH, Hollerithallee 17, 30419 Hannover, Germany \\ 5. Laser Zentrum Hannover e.V., Hollerithallee 8, 30419 Hannover, Germany
}

High repetition rate, ultrabroadband light sources in the near infrared spectral region have gained considerable research interest and emerged as a powerful basis for various applications, such as time-resolved spectroscopy, attoscience, and frequency comb generation [1]. Kerr-lens mode locked (KLM) Ti:sapphire lasers oscillators remain the major workhorse of the research field due to the remarkable spectral bandwidth of this gain material. However, these systems suffer limitation in terms of power scaling mainly owing to unavoidable heat load in the laser crystal. Alternatively, optical parametric oscillators (OPOs) have rapidly developed over the last decade and are a promising candidate to generate high power broadband tunable radiations. However, the pulse forming dynamics in OPOs is more complicated and so far limited the achievable pulse duration to $13 \mathrm{fs}$ [2]. In this work, by introducing a breathing pulse dispersion management scheme, we report the first experimental demonstration of a KLM non-collinear OPO (KLM-NOPO) that emits an ultrabroadband signal spanning from $700 \mathrm{~nm}$ to $900 \mathrm{~nm}$ at the $-10 \mathrm{~dB}$ level which would support sub-10 fs pulse durations.

The schematic of the proposed KLM-NOPO is presented in Fig. 1(a). The OPO is pumped by a frequencydoubled home-built $\mathrm{Yb}$-fiber laser. The pump laser has an average power of $7 \mathrm{~W}$ at a repetition rate of $50.2 \mathrm{MHz}$ with a FWHM pulse duration 270 fs and a central wavelength $520 \mathrm{~nm}$. The KLM-NOPO is configured as a double foci ring cavity. The $2 \mathrm{~mm}$ BBO crystal in one focus serves as the parametric gain medium, while a $1.7 \mathrm{~mm}$ Ti:sapphire crystal used as Kerr-medium is placed in another focus. The whole cavity is equipped with double-chirped mirrors (DCMs) that support a flat spectral phase over 600 1200 nm [3]. A pair of $\mathrm{BaF}_{2} \mathrm{wedges}$ is employed to fine adjust the cavity dispersion, and accordingly the overall dispersion of the cavity is close to zero. Note that a strong unbalanced dispersion between two foci is realized in our setup, leading to pulse breathing in one round trip. To this end, a long positive dispersed signal pulse as expected for a good temporal overlap in the BBO together with a short transform limited pulse in the Ti:sapphire is realized. For synchronous pumping, the cavity length is set to match twice the pump lasers repetition rate.

The "magic angle" of $2.5^{\circ}$ between the pump beam and the signal beam in non-collinear pointing vector walk-off compensation geometry is selected where ultra-broadband phase matching from 650 to $1200 \mathrm{~nm}$ enables broadband operation. By optimizing the position of the Kerr medium, the KLM NOPO can be triggered. As can be seen in Fig. 1(b), this broadband output spectrum covering 670 920 nm provides strong evidence for mode-locked NOPO. This bandwidth results in a transform limited pulse duration of $8.5 \mathrm{fs}$. Up to $420 \mathrm{~mW}$ of output power is measured with the maximum pump power of $7 \mathrm{~W}$. A stable mode-locking performance of our KLM NOPO is indicated by the clean radio-frequency spectrum of the signal (see Fig. 1(c)). In conclusion, the proposed dispersion managed KLM NOPO can generate a stable pulse with an ultra-broadband spectrum capable of handling sub-10 fs pulses. The great potential for power scaling and the flexibility in wavelength of this novel KLM NOPO open the door for the next generation of few-cycle high power laser oscillators.
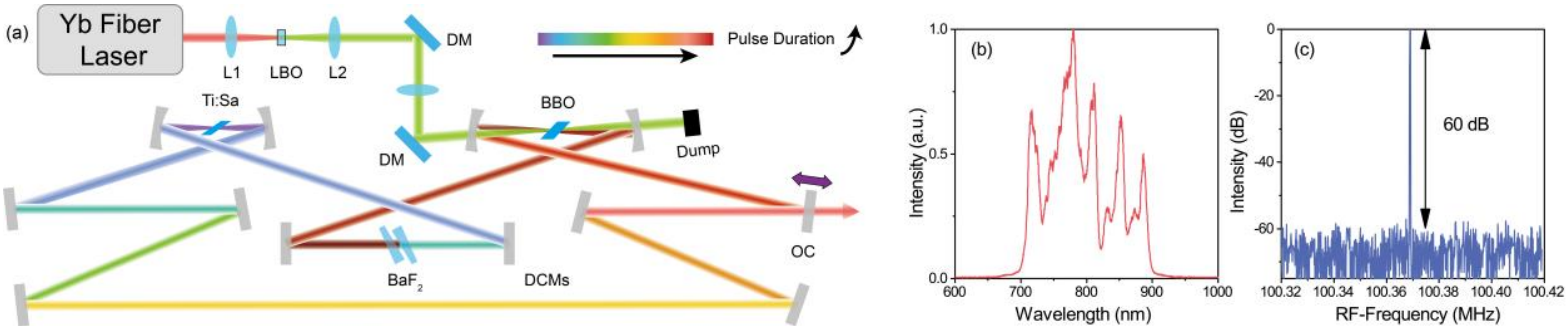

Fig. 1 (a) Schematics illustration of the experimental set-up. L1 and L2: lens; DM: dichroic mirror; DCMs: doublechirped mirror pairs; OC: output coupler. (b) Measured output spectrum (c) Radio-frequency spectrum over a 100 kHz span with $100 \mathrm{~Hz}$ resolution.

\section{References}

[1] S. A. Diddams, L. Hollberg, and V. Mbele, "Molecular fingerprinting with the resolved modes of a femtosecond laser frequency comb," Nature 445, 627-630 (2007).

[2] G. M. Gale, M. Cavallari, T. J. Driscoll, and F. Hache, "Sub-20-fs tunable pulses in the visible from an 82-MHz optical parametric oscillator," Opt. Lett. 20, 1562 (1995).

[3] S. Rausch, T. Binhammer, A. Harth, J. Kim, R. Ell, F. X. Kärtner, and U. Morgner, "Controlled waveforms on the single-cycle scale from a femtosecond oscillator," Opt. Express 16, 9739-9745 (2008) 